\title{
Parameters Design for Logarithmic Quantizer Based on Zoom Strategy
}

\author{
Jingjing Yan \\ College of Electrical Engineering, Henan University of Technology, Zhengzhou 450052, China \\ Correspondence should be addressed to Jingjing Yan; yanjingjing2009@163.com
}

Received 30 December 2016; Revised 8 February 2017; Accepted 12 February 2017; Published 27 February 2017

Academic Editor: Haibo Du

Copyright (c) 2017 Jingjing Yan. This is an open access article distributed under the Creative Commons Attribution License, which permits unrestricted use, distribution, and reproduction in any medium, provided the original work is properly cited.

\begin{abstract}
This paper is concerned with the problem of designing suitable parameters for logarithmic quantizer such that the closed-loop system is asymptotic convergent. Based on zoom strategy, we propose two methods for quantizer parameters design, under which it ensures that the state of the closed-loop system can load in the invariant sets after some certain moments. Then we obtain that the quantizer is unsaturated, and thus the quantization errors are bounded under the time-varying logarithm quantization strategy. On that basis, we obtain that the closed-loop system is asymptotic convergent. A benchmark example is given to show the usefulness of the proposed methods, and the comparison results are illustrated.
\end{abstract}

\section{Introduction}

With the continuous improvement of the communication capability and reliability of the network, the data in the control system is transmitted through the network increasingly. The network bandwidth, although it may be very large, is always limited, so the data quantization is inevitable. At present, zoom strategy proposed in $[1,2]$ is a popular method in quantized control system.

Based on zoom strategy, literature [3] analyzes the stabilization problem for linear systems with multidimensional state and one-dimensional input. The main contribution of that paper is the trade-off between the quantized controller complexity and the system performance. Literature [4] proposes a unified framework to describe both the network conditions and the state quantization of linear systems. A model describing the nonideal network conditions and input/output state quantization is given by [5], and the problem of the quantized output feedback controller is designed there to asymptotically stabilize the closed-loop system. Hereafter, zoom strategy is widely used in quantized feedback stabilization problem.

As for the system affected by data quantization and timedelay, literature [6] proves that under some conditions, the closed-loop system can be global asymptotically stabilized via a dynamic quantization strategy by integral mean value theorem. Literature [7] designs an optimal dynamic quantizer which is able to minimize the maximum output error between the quantized system and unquantized systems. Supposing that the quantizer is to be saturated, two types of quantizer are discussed in [8] such that the state of the closedloop system starting from a neighborhood of the origin exponentially converges to a bounded region. If the closed-loop system is affected by data quantization and packet dropout, we study the quantized stabilization of linear discrete-time systems and discrete-time and continuous-time fuzzy systems, respectively, in [9] and [10]. Assuming that the system is relating to quantization and disturbance, the related results can be shown in [11-13]. In literature [11], the authors propose two specific control strategies that yield the input-to-state stability of the closed-loop system based on quantized state. When the sampled state/output can only be obtained by the controller, literature [12] designs the full state feedback controller and the output feedback controller to stabilize the system. We generalize the results of [12] to the case that the system is also affected by packet dropout in [13]. For the stabilization problem of the closed-loop system affected by quantization and saturation, literature $[8,14]$ gets some results.

Zoom strategy is also combined with other methods to stabilize the closed-loop system, such as sliding-mode control 
method [15], Kalman filter [16], scheduling protocol [17], and event-triggered control [18-20]. Furthermore, it is used to stabilize different kinds of systems, such as switched system [21-24], multiagent system [25, 26], and interconnected system [27]. More related works can be seen in review of literature $[28,29]$.

In summary, zoom strategy is a popular method to adjust the quantizer parameters, especially for uniform quantizer, such that the quantized control system is stable. As we know, logarithmic quantizer is another kind of commonly used quantizer $[30,31]$. Compared with uniform quantizer, logarithmic quantizer has better performance near the origin. Consider this advantage, some papers adopt logarithmic quantization method to quantize the system state or output. Recent results include literature [32-36]. However, most of the existing papers assume that the parameters of the logarithmic quantizer are given previously. Few articles study the parameters design for logarithmic quantizer, which is the main research of this paper.

In this paper, we will propose two design methods for the parameters of the logarithmic quantizer. By zoom strategy, we design the logarithmic quantizer parameters including $v_{0}, \delta$, and quantization density $\rho$, which determine the saturation boundary, dead zone, and the quantization intervals of the quantizer. Under the parameters designed, we guarantee the unsaturation of the quantizer and the asymptotic convergence of the closed-loop system.

The rest of this paper is organized as follows. We describe the problem discussed here in Section 2. Two design methods for the parameters of the logarithmic quantizer are illustrated in Sections 3 and 4, respectively. A well-known benchmark example is adopted in Section 5 to show the effectiveness of the design methods, and their comparison is also illustrated there. Finally, some conclusions are given in Section 6.
Notation. $\mathbb{R}^{n}$ denotes the $n$-dimensional Euclidean space. $\mathbb{R}^{+}$and $\mathbb{N}$ denote the set of positive real numbers and positive integers, respectively. We denote by $\|\cdot\|$ the standard Euclidean norm in $\mathbb{R}^{n}$ and the corresponding induced matrix norm in $\mathbb{R}^{n \times n} \cdot \lambda_{\max }(P)$ and $\lambda_{\text {min }}(P)$ denote the maximum and minimum eigenvalue of matrix $P$, respectively. $A^{\top} \in \mathbb{R}^{m \times n}$ denotes the transposed of matrix $A \in \mathbb{R}^{n \times m}$. The signal $\lceil y\rceil$ indicates the least integer not less than $y . \mathbf{0}_{n \times 1}$ denotes zero vector with dimension $n \times 1$.

\section{Problem Formulation}

This paper considers the following linear time-invariant discrete systems:

$$
x(k+1)=A x(k)+B u(k)
$$

where $x \in \mathbb{R}^{n}$ is the state vector, $u \in \mathbb{R}^{m}$ is the control input, and $A$ and $B$ are constant matrices with proper dimensions. In the following, we assume that the open-loop system is unstable; that is, $\|A\|>1$.

Assuming that the network is located at the sensor side, thus the controller can only receive the quantized values of the system states due to the limited bandwidth. Hence the controller can be illustrated as

$$
u(k)=K Q(x(k))
$$

where $K$ is the feedback matrix to be designed and $Q(\cdot)$ is a logarithmic quantizer defined as

$$
Q(x(k))=\left[q\left(x_{1}(k)\right), \ldots, q\left(x_{n}(k)\right)\right]^{\top} .
$$

Suppose that the quantization level of the quantizer is equal to $2 N+1, N \in \mathbb{N}$. For any $i \in\{1,2, \ldots, n\}$, if $k \in\left[k_{j}, k_{j+1}\right)$, $j \in \mathbb{N} \cup\{0\}$, where $k_{j}$ will be designed below, we define

$$
q\left(x_{i}(k)\right)= \begin{cases}v_{j} & \text { if } x_{i}(k) \in\left[\frac{1}{1+\delta} v_{j},+\infty\right) \\ v_{j+m} & \text { if } x_{i}(k) \in\left[\frac{1}{1+\delta} v_{j+m}, \frac{1}{1-\delta} v_{j+m}\right), m \in\{1,2, \ldots, N-1\} \\ 0 & \text { if } x_{i}(k) \in\left[0, \frac{1}{1+\delta} v_{j+N-1}\right) \\ -q\left(-x_{i}(k)\right) & \text { if } x_{i}(k) \in(-\infty, 0],\end{cases}
$$

with $\delta \in(0,1)$ and $v_{j+1}=\rho v_{j}, v_{0}>0$, in which the quantization density $\rho$ is given by $\rho=(1-\delta) /(1+\delta) \epsilon$ $(0,1)$. Obviously, $\delta, \rho$, and $v_{0}$ are the critical parameters of the logarithmic quantizer, which are assumed to be undetermined.

The aim of this paper is to design the quantizer parameters $\delta, \rho$, and $v_{0}$ based on zoom strategy to ensure the unsaturation of the quantizer; that is, $\|x(k)\| \leq(1 /(1-\delta)) v_{j}$, $k \in\left[k_{j}, k_{j+1}\right), j \in \mathbb{N} \cup\{0\}$. From which we can see that the quantization errors are bounded and the system state tends to zero when $k$ tends to infinity according to the definition of $v_{j}$. The main innovation of this paper is that the zoom strategy, which is always adopted to discuss the properties of the uniform quantizer, is used here to determine the parameters of the logarithmic quantizer.

In what follows, we will propose two methods for quantizer parameters design and compare them in simulation example. 


\section{First Method for Quantizer Parameters Design}

If there exist a positive-definite matrix $P$ and a feedback matrix $K$ such that

$$
\Pi:=P-(A+B K)^{\top} P(A+B K)>0,
$$

that is, the system can be stabilized by standard state feedback, then the asymptotic convergence of the closed-loop system can be guaranteed by the quantizer parameters designed in the following theorem.

Theorem 1. For any given matrices $K, P$, and $\Pi$ defined by (5) and the quantization level $2 N+1$, we select quantizer parameters $\delta$ and $\rho$ satisfying

$$
\rho=\sqrt{\frac{\lambda_{\max }(P)}{\lambda_{\min }(P)}} \Upsilon \sqrt{n} \delta(1+\varepsilon)<1,
$$

where $\varepsilon$ is an arbitrary given positive constant and $\Upsilon$ is defined by $\Upsilon=2\|B K P(A+B K)\| / \lambda_{\min }(\Pi)$. Given a constant $\eta \in \mathbb{N}$, if $\delta$ and $\rho$ selected above satisfy the inequalities $\delta \geq \rho^{N}$ and $\rho^{\eta}<\|A\|^{-1}$, then we have $\|x(k)\| \leq(1 /(1-\delta)) v_{j}$ for any $k \in$ $\left[k_{j}, k_{j+1}\right), j \in \mathbb{N} \cup\{0\}$, where $k_{0}$ and $v_{0}$ are determined by (9) below and $k_{j+1}:=k_{j}+\tau:=k_{j}+\lceil\widetilde{\tau}\rceil$ with

$$
\tilde{\tau}=\frac{\lambda_{\min }(P)-\lambda_{\max }(P)(\Upsilon \sqrt{n}(1+\varepsilon) \delta)^{2}}{\lambda_{\min }(\Pi)(\Upsilon \sqrt{n} \delta)^{2} \varepsilon(1+\varepsilon)} .
$$

Hence we have $\lim _{k \rightarrow \infty} x(k)=\mathbf{0}_{n \times 1}$; that is, the closed-loop system is asymptotic convergent.

Remark 2. If the bandwidth of the network is large enough such that $N \geq \ln \delta / \ln \rho$, then the condition $\delta \geq \rho^{N}$ can always be guaranteed. Moreover, the inequality $\rho^{\eta}<\|A\|^{-1}$ must be ensured if $\eta$ is selected large enough based on $\rho<1$. Comprehensively, if the bandwidth of the network and the constant $\eta$ are set suitably, we can always find the quantizer parameters satisfying the conditions of the above theorem.

Remark 3. Based on the relationship of $\rho$ and $\delta$, that is, $\rho=$ $(1-\delta) /(1+\delta)$, we can determine their values by the equality (6).

\section{Proof.}

Stage 1 (zooming-out). In this stage, the system is as openmode; that is, $x(k+1)=A x(k)$. The aim of this stage is to determine the value of $v_{0}$ and a moment where the system state is unsaturated.

For any given constants $\widetilde{v}_{0} \in \mathbb{R}^{+}, \eta \in \mathbb{N}$, and $\rho$ defined by (6), we set $\widetilde{v}(k), \forall k \in \mathbb{N}$, as

$$
\begin{gathered}
\widetilde{v}(k+1)=\frac{1}{\rho^{\eta}} \widetilde{v}(k), \\
\widetilde{v}(0)=\widetilde{v}_{0} .
\end{gathered}
$$

If $\rho$ satisfies $\rho^{\eta}<\|A\|^{-1}<1$, then there must exist a moment $k_{0}$ such that

$$
\left\|x\left(k_{0}\right)\right\| \leq \sqrt{\frac{\lambda_{\text {min }}(P)}{\lambda_{\text {max }}(P)}} \frac{\widetilde{v}\left(k_{0}\right)}{1-\delta}:=\sqrt{\frac{\lambda_{\text {min }}(P)}{\lambda_{\text {max }}(P)}} \frac{v_{0}}{1-\delta},
$$

and thus

$$
\begin{aligned}
& x\left(k_{0}\right) \in R_{0} \\
& \quad:=\left\{x(k): x^{\top}(k) P x(k) \leq\left(\frac{v_{0}}{1-\delta}\right)^{2} \lambda_{\text {min }}(P)\right\} .
\end{aligned}
$$

Remark 3. The definition of $\widetilde{v}(k), \forall k \in \mathbb{N} \cup\{0\}$, is to ensure that it can be represented as $\widetilde{v}(k)=\rho^{m} \widetilde{v}_{0}$, where $m=l \eta, l \in \mathbb{N} \cup\{0\}$. On the basis of that, we can ensure that $\widetilde{v}(k), \forall k \in \mathbb{N} \cup\{0\}$, is the quantized value of the logarithmic quantizer.

Stage 2 (zooming-in). Let the zooming-out stage finish at the moment $k_{0}$ and the system is transferred to closed-mode. The purpose of this stage is to design the quantizer parameters and $k_{j}, j \in \mathbb{N} \cup\{0\}$, such that $\|x(k)\| \leq(1 /(1-\delta)) v_{j}$ for any $k \in\left[k_{j}, k_{j+1}\right)$. To this end, we first illustrate that $R_{0}$ is an invariant set.

If $x(k) \in R_{0}$, then $\|x(k)\| \leq(1 /(1-\delta)) v_{0}$. Thus we obtain $\left|x_{i}(k)\right| \leq(1 /(1-\delta)) v_{0}$ which results in that

$$
\begin{aligned}
& \left|q\left(x_{i}(k)\right)-x_{i}(k)\right| \\
& \leq \max \left\{\left(\frac{1}{1-\delta}-1\right) v_{0},\left(1-\frac{1}{1+\delta}\right) v_{0}, \frac{1}{1+\delta} v_{N-1}\right\} \\
& =\frac{\delta}{1-\delta} v_{0},
\end{aligned}
$$

where the last equality is obtained by $\delta \geq \rho^{N}$. Hence, we get

$$
\|e(k)\| \leq \sqrt{n} \frac{\delta}{1-\delta} v_{0},
$$

with $e(k):=Q(x(k))-x(k)$.

Let Lyapunov function as $V(k)=x^{\top}(k) P x(k)$, then it is easy to get

$$
\begin{aligned}
\Delta V( & x(k))=x^{\top}(k+1) P x(k+1)-x^{\top}(k) P x(k) \\
= & x^{\top}(k)\left[(A+B K)^{\top} P(A+B K)-P\right] x(k) \\
& +2 e^{\top}(k)(B K)^{\top} P(A+B K) x(k) \\
\leq & -\lambda_{\min }(\Pi)\|x(k)\|^{2} \\
& +2\|B K P(A+B K)\|\|x(k)\| \sqrt{n} \frac{\delta}{1-\delta} v_{0} \\
= & -\lambda_{\min }(\Pi)\|x(k)\|\left(\|x(k)\|-\Upsilon \sqrt{n} \frac{\delta}{1-\delta} v_{0}\right) .
\end{aligned}
$$

For an arbitrary given positive constant $\varepsilon$, if

$$
\|x(k)\| \geq \Upsilon \sqrt{n} \frac{\delta}{1-\delta} v_{0}(1+\varepsilon),
$$


then we get

$$
\Delta V(x(k)) \leq-\lambda_{\min }(\Pi)\|x(k)\| \Upsilon \sqrt{n} \frac{\delta}{1-\delta} v_{0} \varepsilon .
$$

Moreover, by (13) we know that the set $\mathbf{B}$, defined by

$$
\mathbf{B}:=\left\{x(k) \mid x(k) \leq \Upsilon \sqrt{n} \frac{\delta}{1-\delta} v_{0}\right\},
$$

is an invariant set. Define $\widetilde{\mathbf{B}}$ as

$$
\begin{aligned}
\widetilde{\mathbf{B}} & :=\left\{x(k) \mid x^{\top}(k) P x(k)\right. \\
& \left.\leq \lambda_{\max }(P)\left(\Upsilon \sqrt{n} \frac{\delta}{1-\delta} v_{0}\right)^{2}\right\},
\end{aligned}
$$

and it is obvious that $\widetilde{\mathbf{B}} \supset \mathbf{B}$. If $\delta$ selected satisfies the following inequality:

$$
\sqrt{\frac{\lambda_{\max }(P)}{\lambda_{\min }(P)}} \Upsilon \sqrt{n} \delta(1+\varepsilon)<1
$$

we can see that $R_{0} \supset \widetilde{\mathbf{B}} \supset \mathbf{B}$, and thus $R_{0}$ is an invariant set.

Next, we will show that there exists a moment $k_{1}:=k_{0}+\tau$ such that $\left\|x\left(k_{1}\right)\right\| \leq(1 /(1-\delta)) v_{1}$. Let

$$
\rho=\sqrt{\frac{\lambda_{\max }(P)}{\lambda_{\min }(P)}} \Upsilon \sqrt{n} \delta(1+\varepsilon),
$$

and we can claim that

$$
x^{\top}\left(k_{1}\right) P x\left(k_{1}\right) \leq \lambda_{\max }(P)\left(\Upsilon \sqrt{n} \frac{\delta}{1-\delta} v_{0}(1+\varepsilon)\right)^{2} \text {. }
$$

In fact, if (20) does not hold, we get

$$
x^{\top}\left(k_{1}\right) P x\left(k_{1}\right)>\lambda_{\max }(P)\left(\Upsilon \sqrt{n} \frac{\delta}{1-\delta} v_{0}(1+\varepsilon)\right)^{2} \text {. }
$$

Consider that the set $\widetilde{R}_{1}$, defined as

$$
\begin{aligned}
\widetilde{R}_{1} & :=\left\{x(k): x^{\top}(k) P x(k)\right. \\
& \left.\leq \lambda_{\max }(P)\left(\Upsilon \sqrt{n} \frac{\delta}{1-\delta} v_{0}(1+\varepsilon)\right)^{2}\right\},
\end{aligned}
$$

is an invariant set based on $\widetilde{R}_{1} \supset \mathbf{B}$. Combined with (21) we know that

$$
x^{\top}(k) P x(k)>\lambda_{\max }(P)\left(\Upsilon \sqrt{n} \frac{\delta}{1-\delta} v_{0}(1+\varepsilon)\right)^{2}
$$

holds for any $k \in\left[k_{0}, k_{1}\right]$. Hence we get

$$
\|x(k)\|>\Upsilon \sqrt{n} \frac{\delta}{1-\delta} v_{0}(1+\varepsilon),
$$

for any $k \in\left[k_{0}, k_{1}\right]$. Similar to the analysis of (13)-(15), we get

$$
\begin{aligned}
\Delta V & \left(x\left(k_{0}+\tau-i\right)\right) \\
= & x^{\top}\left(k_{0}+\tau-i+1\right) P x\left(k_{0}+\tau-i+1\right) \\
& -x^{\top}\left(k_{0}+\tau-i\right) P x\left(k_{0}+\tau-i\right) \\
\leq & -\lambda_{\min }(\Pi)\left\|x\left(k_{0}+\tau-i\right)\right\| \Upsilon \sqrt{n} \frac{\delta}{1-\delta} v_{0} \varepsilon \\
< & -\lambda_{\min }(\Pi)\left(\Upsilon \sqrt{n} \frac{\delta}{1-\delta} v_{0}\right)^{2} \varepsilon(1+\varepsilon),
\end{aligned}
$$

for any $i \in\{1,2, \ldots, \tau\}$. Hence it is obtained that

$$
\begin{aligned}
x^{\top} & \left(k_{0}+\tau\right) P x\left(k_{0}+\tau\right)-x^{\top}\left(k_{0}\right) P x\left(k_{0}\right) \\
<- & \lambda_{\min }(\Pi)\left(\Upsilon \sqrt{n} \frac{\delta}{1-\delta} v_{0}\right)^{2} \varepsilon(1+\varepsilon) \tau \\
\leq & -\lambda_{\min }(\Pi)\left(\Upsilon \sqrt{n} \frac{\delta}{1-\delta} v_{0}\right)^{2} \varepsilon(1+\varepsilon) \tilde{\tau} \\
= & \lambda_{\max }(P)(\Upsilon \sqrt{n}(1+\varepsilon) \delta)^{2}\left(\frac{v_{0}}{1-\delta}\right)^{2} \\
& -\lambda_{\min }(P)\left(\frac{v_{0}}{1-\delta}\right)^{2} .
\end{aligned}
$$

However, (10) and (21) give that

$$
\begin{aligned}
x^{\top}\left(k_{0}+\tau\right) P x\left(k_{0}+\tau\right)-x^{\top}\left(k_{0}\right) \operatorname{Px}\left(k_{0}\right) \\
>\lambda_{\max }(P)(\Upsilon \sqrt{n}(1+\varepsilon) \delta)^{2}\left(\frac{v_{0}}{1-\delta}\right)^{2} \\
-\lambda_{\min }(P)\left(\frac{v_{0}}{1-\delta}\right)^{2},
\end{aligned}
$$

which contradicts formula (26). Therefore, the claim (20) holds; that is,

$$
\begin{aligned}
x^{\top}\left(k_{1}\right) P x\left(k_{1}\right) & \leq \lambda_{\max }(P)\left(\Upsilon \sqrt{n} \frac{\delta}{1-\delta} v_{0}(1+\varepsilon)\right)^{2} \\
& =\lambda_{\min }(P)\left(\frac{\rho v_{0}}{1-\delta}\right)^{2} \\
& =\lambda_{\min }(P)\left(\frac{v_{1}}{1-\delta}\right)^{2} .
\end{aligned}
$$

We define the set $R_{1}$ as

$$
R_{1}:=\left\{x(k): x^{\top}(k) P x(k) \leq\left(\frac{v_{1}}{1-\delta}\right)^{2} \lambda_{\min }(P)\right\} .
$$

Similar to the analysis of $R_{0}$, we know that $R_{1}$ is an invariant set. Thus it is easy to see that $x(k) \in R_{1}, \forall k \in\left[k_{1}, k_{2}\right)$. Furthermore, similar analysis gives

$$
\begin{aligned}
x(k) & \in R_{j} \\
:= & \left\{x(k): x^{\top}(k) P x(k) \leq\left(\frac{v_{j}}{1-\delta}\right)^{2} \lambda_{\min }(P)\right\},
\end{aligned}
$$


for any $k \in\left[k_{j}, k_{j+1}\right), j \in \mathbb{N} \cup\{0\}$. So far, we know that $\|x(k)\| \leq v_{j} /(1-\delta), \forall k \in\left[k_{j}, k_{j+1}\right), j \in \mathbb{N} \cup\{0\}$; that is, the quantizer is unsaturated for any $k \in\left[k_{0},+\infty\right)$. Hence, we get $\lim _{k \rightarrow \infty}\|x(k)\|=0$ and thus $\lim _{k \rightarrow \infty} x(k)=\mathbf{0}_{n \times 1}$, which completes the proof.

\section{Second Method for Quantizer Parameters Design}

If there are matrices $P$ and $K$ such that the following inequality holds

$$
\Lambda:=P-2(A+B K)^{\top} P(A+B K)>0,
$$

then the asymptotic convergence of the closed-loop system can also be obtained by the quantizer parameters designed in Theorem 4.

Theorem 4. For any given matrices $K, P$, and $\Lambda$ defined by (31) and the quantization level $2 N+1$, quantizer parameters $\delta$ and $\rho$ are selected satisfying

$$
\rho=\sqrt{\frac{\lambda_{\max }(P)}{\lambda_{\min }(P)}} \sqrt{\Gamma(1+\varepsilon)} \sqrt{n} \delta<1,
$$

where $\varepsilon$ is a positive constant and $\Gamma$ is defined by $\Gamma=$ $\left\|(B K)^{\top} P B K\right\| / \lambda_{\min }(\Lambda)$. Given a constant $\eta \in \mathbb{N}$, if $\delta$ and $\rho$ selected above satisfy $\delta \geq \rho^{N}$ and $\rho^{\eta}<\|A\|^{-1}$, then we get $\|x(k)\| \leq(1 /(1-\delta)) v_{j}$ for any $k \in\left[k_{j}, k_{j+1}\right), j \in \mathbb{N} \cup\{0\}$, where $k_{0}$ and $v_{0}$ are determined by (9) and $k_{j+1}:=k_{j}+\omega:=k_{j}+\lceil\widetilde{\omega}\rceil$ with

$$
\widetilde{\omega}=\frac{\lambda_{\min }(P)-\lambda_{\max }(P) \Gamma(1+\varepsilon) n \delta^{2}}{\lambda_{\min }(\Lambda) \Gamma \varepsilon n \delta^{2}} .
$$

Then we have $\lim _{k \rightarrow \infty} x(k)=\mathbf{0}_{n \times 1}$.

Proof.

Stage 1 (zooming-out). The same as the proof of Theorem 1 , we get a moment $k_{0}$ and a positive constant $v_{0}$ such that

$$
\begin{aligned}
& x\left(k_{0}\right) \in \bar{R}_{0} \\
& \quad:=\left\{x(k): x^{\top}(k) P x(k) \leq\left(\frac{v_{0}}{1-\delta}\right)^{2} \lambda_{\min }(P)\right\} .
\end{aligned}
$$

Note that the positive-definite matrix $P$ used here is defined by (31) rather than (5); thus the definitions of the sets $\bar{R}_{0}$ and $R_{0}$ are different from each other.
Stage 2 (zooming-in). If $\delta$ and $\rho$ selected satisfy $\delta \geq \rho^{N}$ and $x(k) \in \bar{R}_{0}, \forall k \in \mathbb{N}$, then we know that $\|e(k)\| \leq \sqrt{n}(\delta /(1-$ $\delta)) v_{0}$ holds, and thus

$$
\begin{aligned}
\Delta V(x(k)) & \\
\leq & x^{\top}(k)\left[2(A+B K)^{\top} P(A+B K)-P\right] x(k) \\
& +e^{\top}(k)(B K)^{\top} P B K e(k) \\
\leq & -\lambda_{\min }(\Lambda)\|x(k)\|^{2} \\
& +\left\|(B K)^{\top} P B K\right\|\left(\sqrt{n} \frac{\delta}{1-\delta} v_{0}\right)^{2} .
\end{aligned}
$$

If

$$
\|x(k)\| \geq \sqrt{\Gamma(1+\varepsilon)} \sqrt{n} \frac{\delta}{1-\delta} v_{0},
$$

then we have

$$
\Delta V(x(k)) \leq-\lambda_{\min }(\Lambda) \Gamma \varepsilon\left(\sqrt{n} \frac{\delta}{1-\delta} v_{0}\right)^{2} .
$$

Due to that the set $\overline{\mathbf{B}}$, defined by

$$
\overline{\mathbf{B}}:=\left\{x(k) \mid x(k) \leq \sqrt{\Gamma} \sqrt{n} \frac{\delta}{1-\delta} v_{0}\right\},
$$

is an invariant one; if we select $\delta$ small enough such that

$$
\sqrt{\frac{\lambda_{\max }(P)}{\lambda_{\min }(P)}} \sqrt{\Gamma(1+\varepsilon)} \sqrt{n} \delta<1,
$$

then we get $\bar{R}_{0} \supset \overline{\mathbf{B}}$ and thus $\bar{R}_{0}$ is an invariant set. Definition of $\rho$ is as in (32) and $k_{1}=k_{0}+\omega$; we claim that

$$
x^{\top}\left(k_{1}\right) P x\left(k_{1}\right) \leq \lambda_{\max }(P) \Gamma(1+\varepsilon) n\left(\frac{\delta}{1-\delta} v_{0}\right)^{2} .
$$

In fact, if (40) does not hold, we know that

$$
x^{\top}(k) P x(k)>\lambda_{\max }(P) \Gamma(1+\varepsilon) n\left(\frac{\delta}{1-\delta} v_{0}\right)^{2},
$$

and thus

$$
\|x(k)\|>\sqrt{\Gamma(1+\varepsilon) n}\left(\frac{\delta}{1-\delta} v_{0}\right)
$$

holds for any $k \in\left[k_{0}, k_{1}\right]$. Based on (37) we get

$$
\Delta V\left(x\left(k_{0}+\omega-i\right)\right)<-\lambda_{\min }(\Lambda) \Gamma \varepsilon\left(\sqrt{n} \frac{\delta}{1-\delta} v_{0}\right)^{2},
$$

for any $i \in\{1,2, \ldots, \omega\}$. Hence, the following inequality can be given

$$
\begin{aligned}
x^{\top} & \left(k_{0}+\tau\right) P x\left(k_{0}+\tau\right)-x^{\top}\left(k_{0}\right) P x\left(k_{0}\right) \\
< & -\lambda_{\min }(\Lambda) \Gamma \varepsilon\left(\sqrt{n} \frac{\delta}{1-\delta} v_{0}\right)^{2} \widetilde{\omega} \\
= & \lambda_{\max }(P) \Gamma(1+\varepsilon) n \delta^{2}\left(\frac{v_{0}}{1-\delta}\right)^{2} \\
& -\lambda_{\min }(P)\left(\frac{v_{0}}{1-\delta}\right)^{2} .
\end{aligned}
$$


But (34) and (41) give that

$$
\begin{gathered}
x^{\top}\left(k_{0}+\tau\right) P x\left(k_{0}+\tau\right)-x^{\top}\left(k_{0}\right) P x\left(k_{0}\right) \\
>\lambda_{\max }(P) \Gamma(1+\varepsilon) n \delta^{2}\left(\frac{v_{0}}{1-\delta}\right)^{2} \\
-\lambda_{\min }(P)\left(\frac{v_{0}}{1-\delta}\right)^{2}
\end{gathered}
$$

which results in a contradiction. Thus the claim (40) holds, combined with the definition of $\rho$ which results in

$$
x^{\top}\left(k_{1}\right) P x\left(k_{1}\right) \leq \lambda_{\min }(P)\left(\frac{v_{1}}{1-\delta}\right)^{2} .
$$

Similar to the proof of Theorem 1, we obtain the unsaturation of the quantizer and the asymptotic convergence of the closed-loop system. This completes the proof.

\section{Simulation}

In this section, we adopt a well-known benchmark example to illustrate the effectiveness of the main results. Let discretization interval be 0.2 ; we convert the continuoustime linearized model of the benchmark to discrete-time one shown as

$$
x(k+1)=A x(k)+B u(k),
$$

with

$$
\begin{aligned}
& A:=\left[\begin{array}{cccc}
1.4381 & 0.0864 & 0.9830 & -0.5676 \\
-0.0972 & 0.4477 & -0.0553 & 0.1009 \\
0.1047 & 0.4929 & 0.2662 & 0.4775 \\
-0.0437 & 0.3930 & -0.1274 & 0.6201
\end{array}\right], \\
& B:=\left[\begin{array}{cc}
0.0645 & -0.3382 \\
0.7823 & 0.0132 \\
0.5413 & -0.3478 \\
0.4578 & 0.0507
\end{array}\right] .
\end{aligned}
$$

Case $1(N=11)$.

Method I. Obviously, the above system is unstable but stabilizable. Thus we can design a feedback matrix $K$ as

$$
K=\left[\begin{array}{cccc}
0.2260 & -0.7118 & 0.1752 & -0.7204 \\
2.6884 & 0.1380 & 2.1328 & -1.1746
\end{array}\right]
$$

and a positive-definite $P$ by LMI toolbox in MATLAB such that the inequality (5) holds. By the definition of matrix $\Pi$, direct calculations result in $\Upsilon=0.9070, \delta=0.1043, \rho=$ 0.8111 , and $\tau=13$, where $\varepsilon$ is selected as 0.5 . Let $\eta=3$; we obtain that $\delta \geq \rho^{N}$ and $\rho^{\eta}<\|A\|^{-1}$ hold. Let $x_{0}=$ $[9,0,10,20]^{\top}$ and $\widetilde{v}_{0}=10$; we get $k_{0}=6$ by Figure 1 , where

$$
M(k):=\sqrt{\frac{\lambda_{\min }(P)}{\lambda_{\max }(P)}} \frac{\widetilde{v}(k)}{1-\delta} .
$$

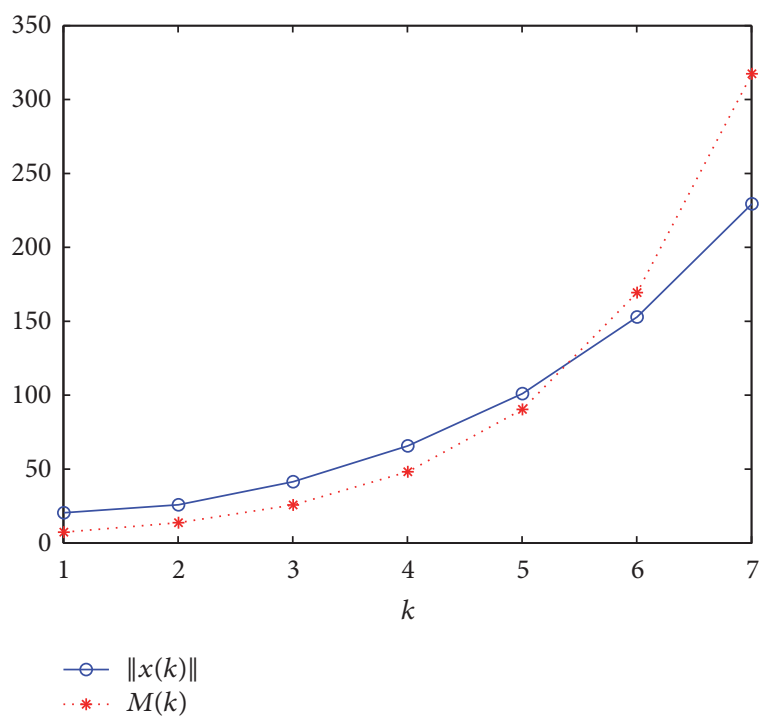

FIGURE 1: Selection of $k_{0}$.

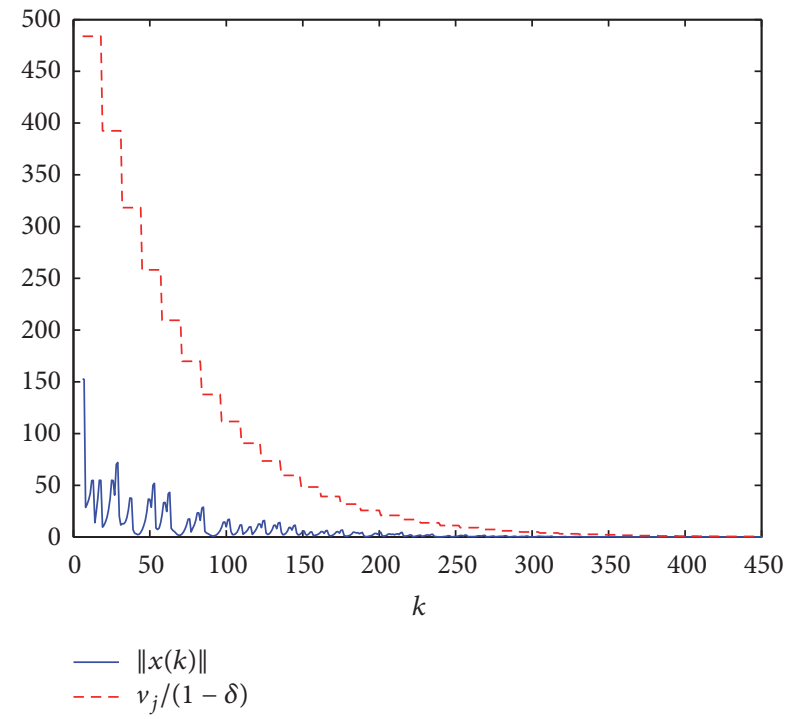

Figure 2: Unsaturation of the quantizer when $N=11$ under method I.

Let $k_{j+1}:=k_{j}+\tau, \forall k \in\left[k_{j}, k_{j+1}\right), j \in \mathbb{N} \cup\{0\}$; we obtain that $\|x(k)\| \leq(1 /(1-\delta)) v_{j}$ for any $k \in\left[k_{j}, k_{j+1}\right)$ and $\lim _{k \rightarrow \infty} x(k)=\mathbf{0}_{n \times 1}$, which are represented in Figures 2 and 3 , respectively.

Method II. In fact, if $K$ and $P$ are selected as the ones in Method I, we know that the matrix $\Lambda$ defined by (31) is positive-definite. If $\varepsilon=0.5$ and $\eta=11$, by simple calculations we get $\Gamma=20.9343, \delta=0.0294$, and $\rho=0.9428$. Obviously, the condition $\rho^{\eta}<\|A\|^{-1}$ is satisfied but $\delta \geq \rho^{N}$ is violated. Hence, Theorem 4 is invalid when $N=11$.

Case $2(N=60)$.

Method $I$. If the matrices $K$ and $P$ and the variables $\varepsilon$ and $\eta$ are selected as the ones in Case 1 , then the corresponding 

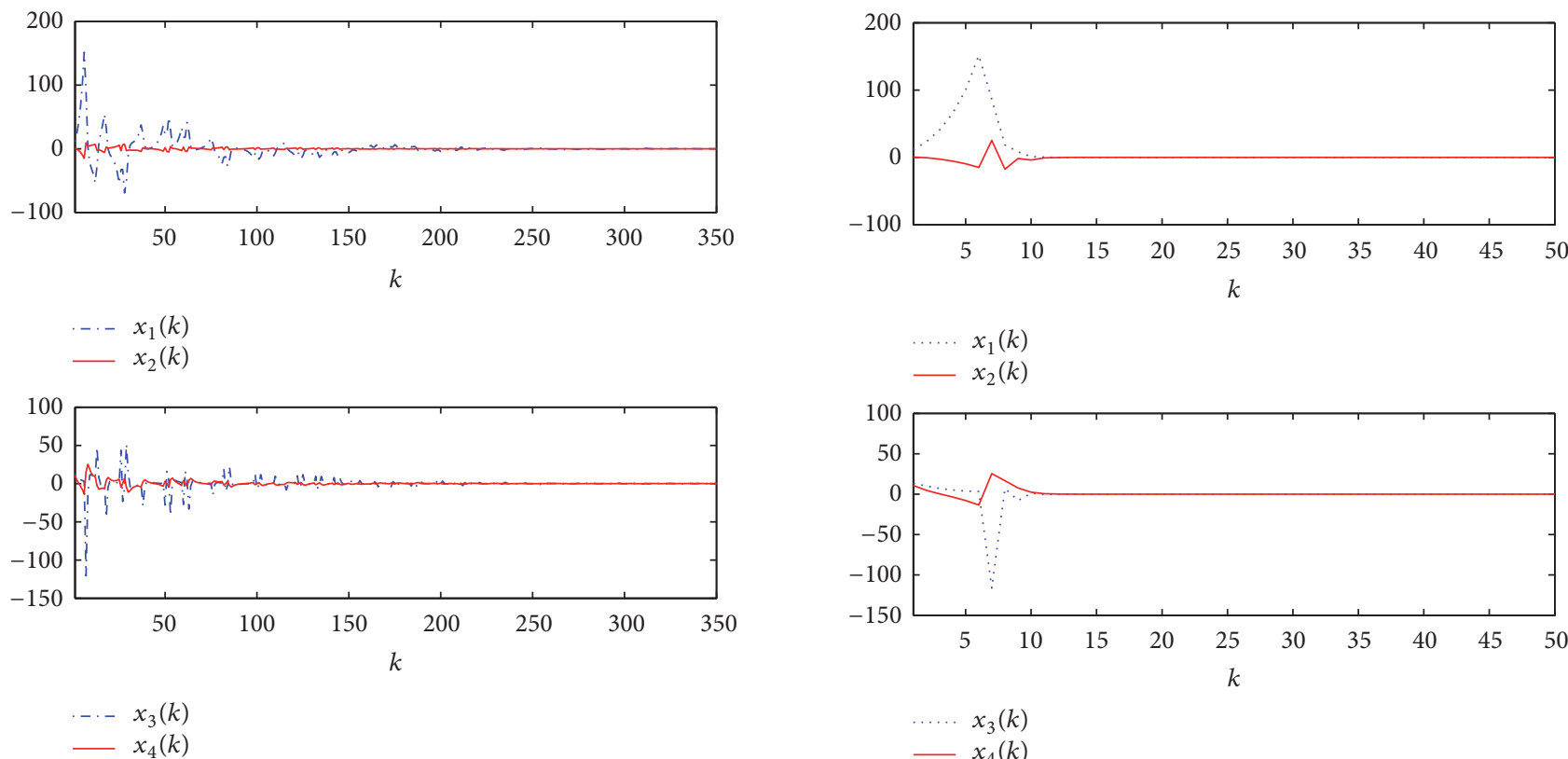

Figure 3: Asymptotic convergence of the system when $N=11$ under method I.

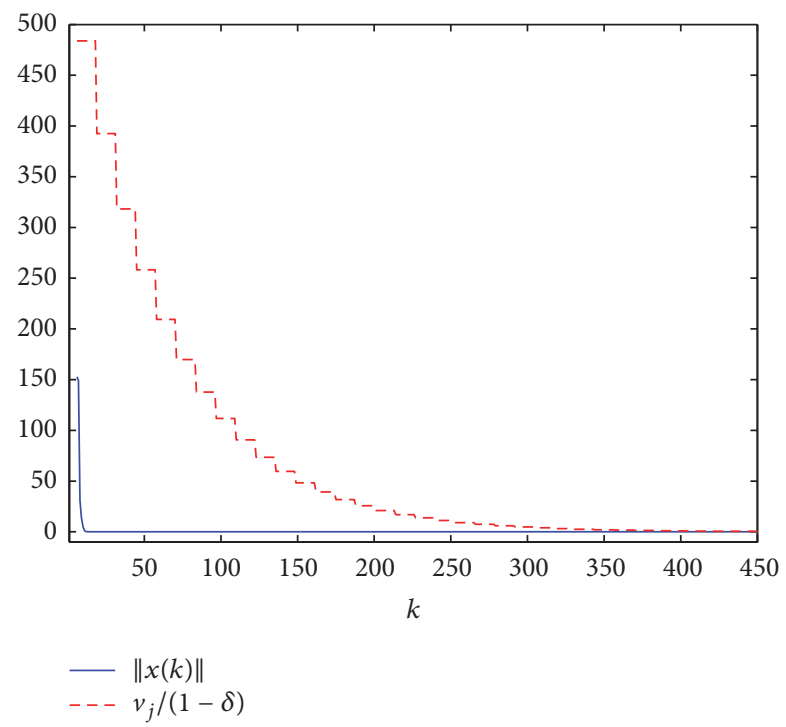

Figure 4: Unsaturation of the quantizer when $N=60$ under method I.

variables calculated are the same as above. Due to $N=$ $60>11$, the conditions on Theorem 1 are satisfied. If $x_{0}=[9,0,10,20]^{\top}$ and $\widetilde{v}_{0}=10$, we also get $k_{0}=$ 6 . The unsaturation of the quantizer and the asymptotic convergence of the closed-loop system are shown in Figures 4 and 5 , respectively.

Method II. If $K, P, \varepsilon$, and $\eta$ are selected as the ones in Case 1, we get that $\Gamma, \delta$, and $\rho$ are the same as above. If $N=60$, then the conditions on Theorem 4 are satisfied. If $x_{0}=[9,0,10,20]^{\top}$ and $\widetilde{v}_{0}=10$, then we get $k_{0}=6$ and $\omega=4$. By Figures 6 and

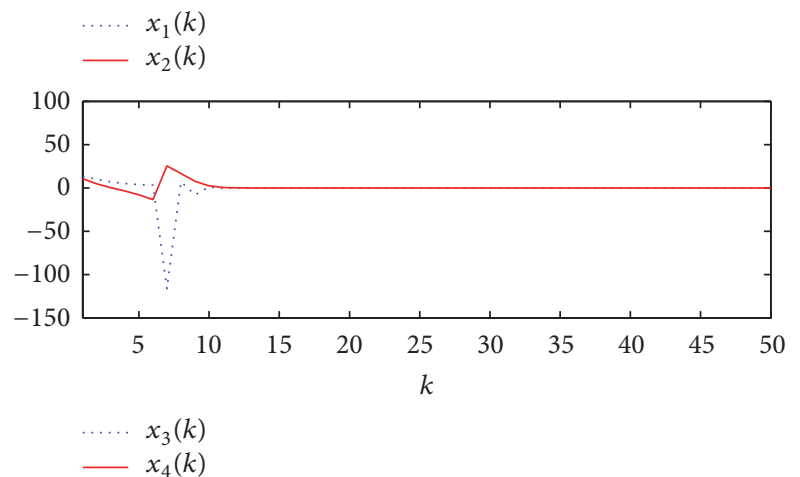

FIGURE 5: Asymptotic convergence of the system when $N=60$ under method I.

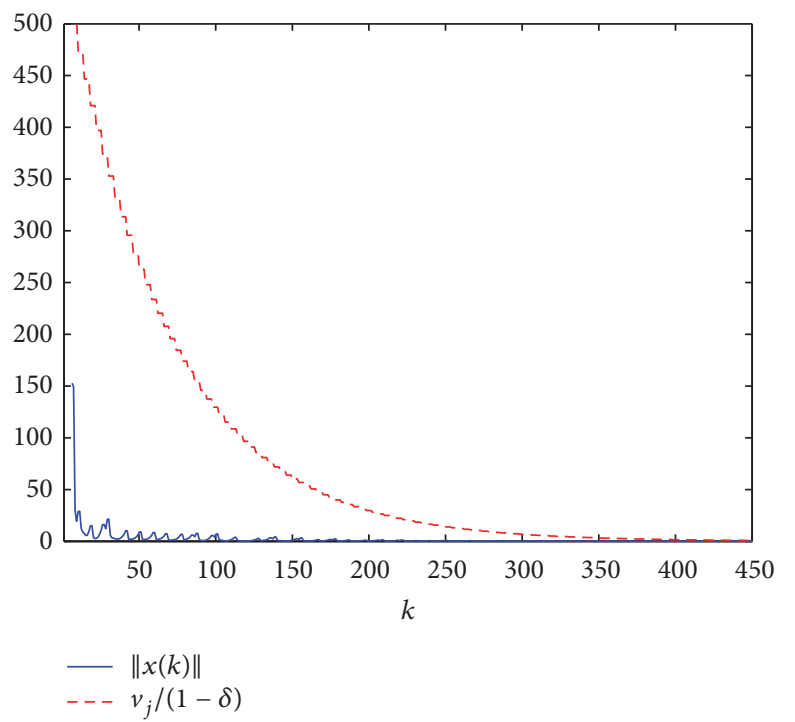

FIGURE 6: Unsaturation of the quantizer when $N=60$ under method II.

7, we know that the quantizer is unsaturated and the closedloop system is asymptotic convergent.

Comparison. From Case 1, we obtain that the first design method has a wider applicability than the second one. When $N$ is large enough, two methods proposed here can guarantee the unsaturation of the quantizer and the asymptotic convergence of the closed-loop system according to the discussions of Case 2. Note that the decreasing period $\omega$ of the second method is less than the one, that is, $\tau$, of the first method. However, the decreasing rate $\rho$ of the second method is 

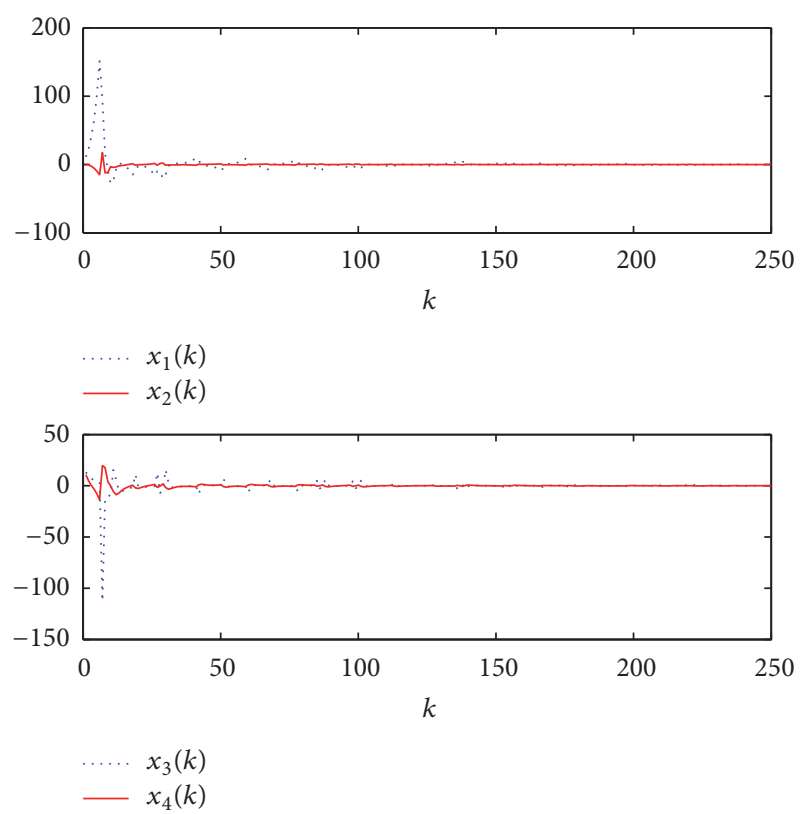

FIgURE 7: Asymptotic convergence of the system when $N=60$ under method II.

larger than the one of the first method. Comprehensively, the convergence speed of the closed-loop system under the second method is slower than the one under the first method. The reason of this is that the second method adopts the following inequality:

$$
E^{T} P F+F^{T} P E \leq E^{T} P E+F^{T} P F
$$

with positive-definite matrix $P$ and matrices $E$ and $F$ to amplify the formula, but the first method avoids it.

\section{Conclusion}

This paper proposed two design methods for logarithmic quantizer parameters. Under both methods, we ensured the unsaturation of the quantizer and the asymptotic convergence of the closed-loop system. Further research includes designing the logarithmic quantizer parameters when the system is affected by network-induced imperfection, like time-delay, packet dropout, and so on.

\section{Competing Interests}

The author declares that he has no competing interests.

\section{Acknowledgments}

The work was supported by the National Natural Science Foundation of China (61403125, 6150020851, and U150410292) and the Natural Science Foundation of Henan Province Education Department (15A413012).

\section{References}

[1] R. W. Brockett and D. Liberzon, "Quantized feedback stabilization of linear systems," Institute of Electrical and Electronics Engineers. Transactions on Automatic Control, vol. 45, no. 7, pp. 1279-1289, 2000.

[2] D. Liberzon, "Hybrid feedback stabilization of systems with quantized signals," Automatica, vol. 39, no. 9, pp. 1543-1554, 2003.

[3] F. Fagnani and S. Zampieri, "Quantized stabilization of linear systems: complexity versus performance," Institute of Electrical and Electronics Engineers. Transactions on Automatic Control, vol. 49, no. 9, pp. 1534-1548, 2004.

[4] C. Peng and Y.-C. Tian, "Networked $H_{\infty}$ control of linear systems with state quantization," Information Sciences, vol. 177, no. 24, pp. 5763-5774, 2007.

[5] E. Tian, D. Yue, and C. Peng, "Quantized output feedback control for networked control systems," Information Sciences. An International Journal, vol. 178, no. 12, pp. 2734-2749, 2008.

[6] D. Liberzon, "Quantization, time delays, and nonlinear stabilization," IEEE Transactions on Automatic Control, vol. 51, no. 7, pp. 1190-1195, 2006.

[7] M. Li, J. Sun, and L. Dou, "Stability of an improved dynamic quantised system with time-varying delay and packet losses," IET Control Theory and Applications, vol. 9, no. 6, pp. 988-995, 2015.

[8] E. Fridman and M. Dambrine, "Control under quantization, saturation and delay: an LMI approach," Automatica. A Journal of IFAC, the International Federation of Automatic Control, vol. 45, no. 10, pp. 2258-2264, 2009.

[9] J. Yan, Y. Xia, B. Liu, and M. Fu, "Stabilisation of quantised linear systems with packet dropout," IET Control Theory \& Applications, vol. 5, no. 8, pp. 982-989, 2011.

[10] J. Yan, Y. Xia, and L. Li, "Stabilization of fuzzy systems with quantization and packet dropout," International Journal of Robust and Nonlinear Control, vol. 24, no. 10, pp. 1563-1583, 2014.

[11] D. Liberzon and D. Nesic, "Input-to-state stabilization of linear systems with quantized state measurements," IEEE Transactions on Automatic Control, vol. 52, no. 5, pp. 767-781, 2007.

[12] Y. Sharon and D. Liberzon, "Input to state stabilizing controller for systems with coarse quantization," IEEE Transactions on Automatic Control, vol. 57, no. 4, pp. 830-844, 2012.

[13] J. Yan and Y. Xia, "Quantized control for networked control systems with packet dropout and unknown disturbances," Information Sciences, vol. 354, pp. 86-100, 2016.

[14] H. Yang, Y. Xia, H. Yuan, and J. Yan, "Quantized stabilization of networked control systems with actuator saturation," International Journal of Robust and Nonlinear Control, vol. 26, no. 16, pp. 3595-3610, 2016.

[15] B. Andrievsky, A. Andrievsky, and I. Zaitceva, "Adaptive zooming strategy in discrete-time implementation of sliding-mode control," in Proceedings of the 1st IFAC Conference on Modelling, Identification and Control of Nonlinear Systems (MICNON '15), pp. 319-326, Saint Petersburg, Russia, June 2015.

[16] Y. Xia, J. Yan, J. Shang, M. Fu, and B. Liu, "Stabilization of quantized systems based on Kalman filter," Control Engineering Practice, vol. 20, no. 10, pp. 954-962, 2012.

[17] K. Liu, E. Fridman, K. H. Johansson, and Y. Xia, "Quantized Control under round-robin communication protocol," IEEE Transactions on Industrial Electronics, vol. 63, no. 7, pp. 44614471, 2016. 
[18] P. Tallapragada and J. Cortes, "Event-triggered stabilization of linear systems under bounded bit rates," IEEE Transactions on Automatic Control, vol. 61, no. 6, pp. 1575-1589, 2016.

[19] Y. Guan, Q.-L. Han, and C. Peng, "Event-triggered quantizeddata feedback control for linear systems," in Proceedings of the IEEE International Symposium on Industrial Electronics (ISIE '13), pp. 1-6, IEEE, Taipei, Taiwan, May 2013.

[20] T. Sun and L. Zhou, "Model-based event-triggered quantized control for linear discrete-time systems," in Proceedings of the 27th Chinese Control and Decision Conference (CCDC '15), pp. 5281-5286, May 2015.

[21] D. Liberzon, "Finite data-rate feedback stabilization of switched and hybrid linear systems," Automatica. A Journal of IFAC, the International Federation of Automatic Control, vol. 50, no. 2, pp. 409-420, 2014.

[22] M. Wakaiki and Y. Yamamoto, "Stability analysis of sampleddata switched systems with quantization," Automatica, vol. 69, pp. 157-168, 2016.

[23] M. Wakaiki and Y. Yamamoto, "Stabilization of switched linear systems with quantized output and switching delays," IEEE Transactions on Automatic Control, 2016.

[24] G. Yang and D. Liberzon, "Finite data-rate stabilization of a switched linear system with unknown disturbance," IFACPapersOnLine, vol. 49, no. 18, pp. 1085-1090, 2016.

[25] Z. Zeng, X. Wang, Z. Zheng, and L. Zhao, "Edge agreement of second-order multi-agent system with dynamic quantization via the directed edge Laplacian," Nonlinear Analysis. Hybrid Systems, vol. 23, pp. 1-10, 2017.

[26] P. Yi and Y. Hong, "Quantized subgradient algorithm and datarate analysis for distributed optimization," IEEE Transactions on Control of Network Systems, vol. 1, no. 4, pp. 380-392, 2014.

[27] S. Dashkovskiy and L. Naujok, "Quasi-ISS/ISDS observers for interconnected systems and applications," Systems \& Control Letters, vol. 77, pp. 11-21, 2015.

[28] Z.-P. Jiang and T.-F. Liu, "Quantized nonlinear control-a survey," Acta Automatica Sinica, vol. 39, no. 11, pp. 1820-1830, 2013.

[29] D. Zhang, P. Shi, Q. Wang, and L. Yu, "Analysis and synthesis of networked control systems: a survey of recent advances and challenges," ISA Transactions, vol. 66, pp. 376-392, 2017.

[30] N. Elia and S. K. Mitter, "Stabilization of linear systems with limited information," Institute of Electrical and Electronics Engineers. Transactions on Automatic Control, vol. 46, no. 9, pp. 1384-1400, 2001.

[31] M. Fu and L. Xie, "The sector bound approach to quantized feedback control," IEEE Transactions on Automatic Control, vol. 50, no. 11, pp. 1698-1711, 2005.

[32] D. Du, B. Qi, M. Fei, and Z. Wang, "Quantized control of distributed event-triggered networked control systems with hybrid wired-wireless networks communication constraints," Information Sciences, vol. 380, pp. 74-91, 2017.

[33] W. Liu, C. Lim, P. Shi, and S. Xu, "Backstepping fuzzy adaptive control for a class of quantized nonlinear systems," IEEE Transactions on Fuzzy Systems, p. 1, 2017.

[34] Y. Wang, W. Ren, and Y. Lu, "Nonfragile $H_{\infty}$ filtering for nonlinear markovian jumping systems with mode-dependent time delays and quantization," Journal of Control Science and Engineering, vol. 2016, Article ID 7167692, 11 pages, 2016.

[35] Y. He, Y.-E. Wang, and X.-M. Li, "Quadratic stabilization for linear time-delay systems with a logarithmic quantizer," Neurocomputing, vol. 173, pp. 1995-2000, 2016.
[36] L. Xing, C. Wen, Y. Zhu, H. Su, and Z. Liu, "Output feedback control for uncertain nonlinear systems with input quantization," Automatica, vol. 65, pp. 191-202, 2016. 


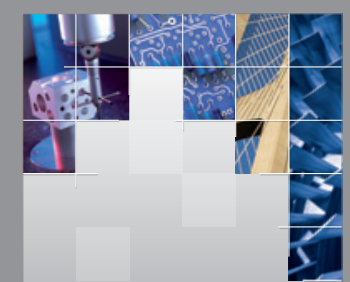

\section{Enfincering}
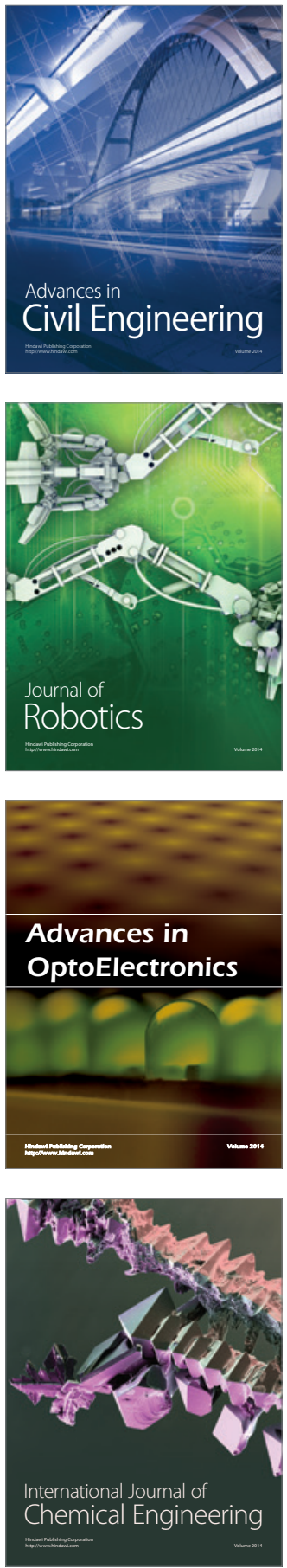

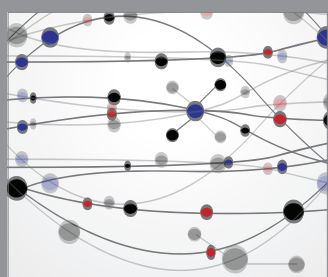

The Scientific World Journal

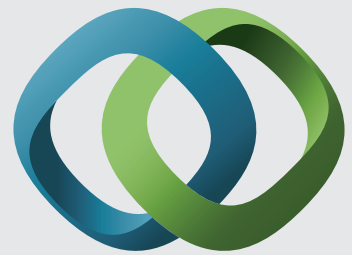

\section{Hindawi}

Submit your manuscripts at

https://www.hindawi.com
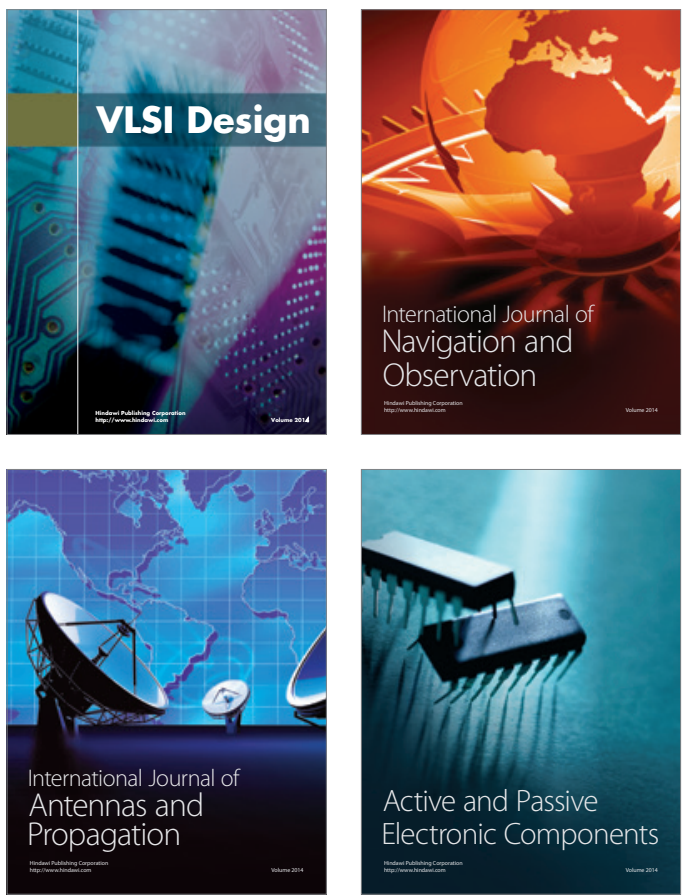
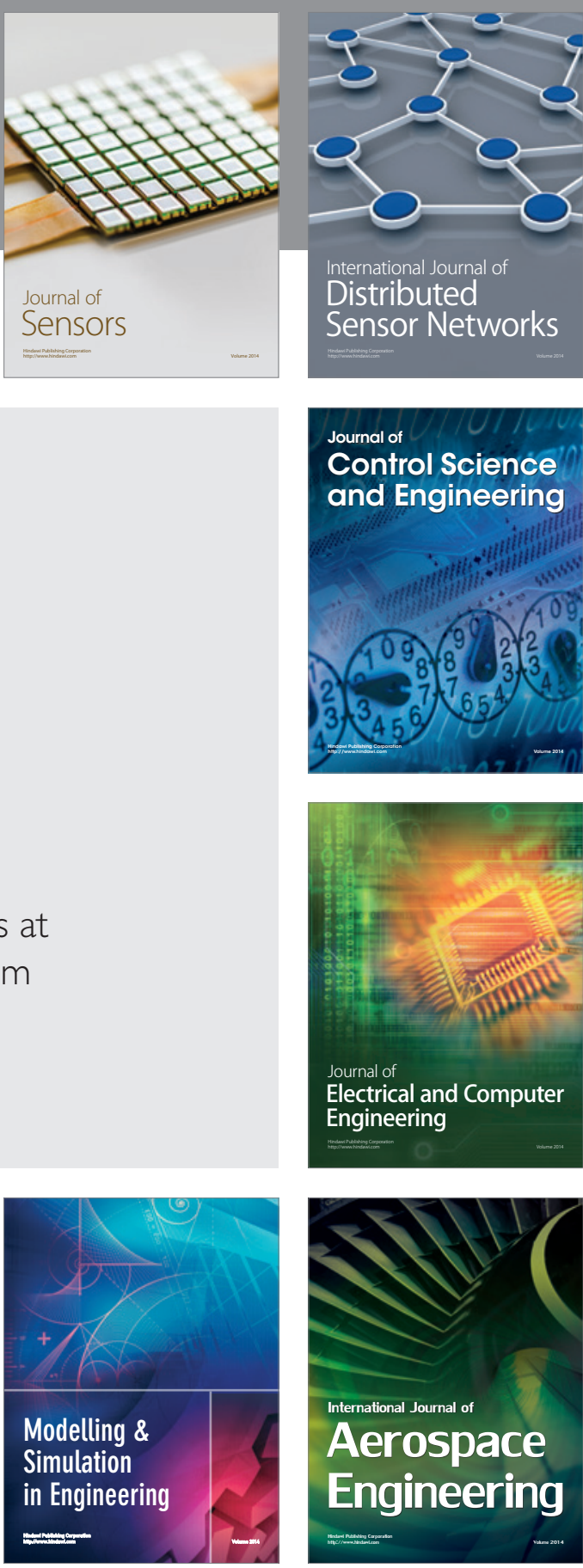

International Journal of

Distributed

Sensor Networks

$-$

Joumal of

Control Science

and Engineering
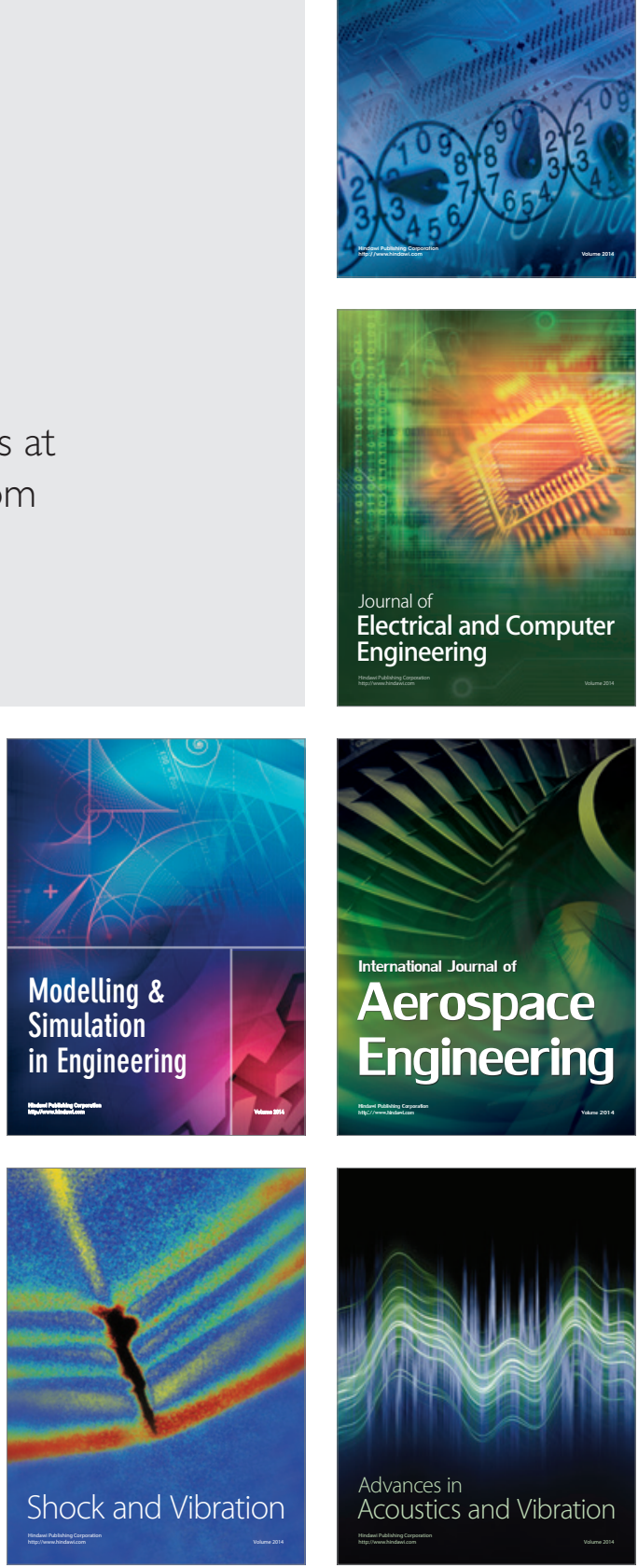